\title{
Analog electro-optical readout of SiPMs for compact, low power ToF PET/MRI
}

\author{
Matthew F Bieniosek ${ }^{1,2,4^{*}}$, Craig S Levin ${ }^{1,2,3,4,5}$ \\ From PSMR14: 3rd Conference in PET/MR and SPECT/MR \\ Kos Island, Greece. 19-21 May 2014
}

${ }^{1}$ Department of Radiology, Stanford University, Stanford, CA 94305, USA
The aim of this work is to demonstrate time of flight (ToF) performance from analog electro-optical transmission of SiPM-based PET detector signals. In electro-optical readout schemes, scintillation signals are converted to near-infrared light by a laser diode and transmitted out of the MRI bore with fiber-optics [1], greatly reducing the PET system's footprint, power consumption, and mutual interference with the MRI.

Our approach uses vertical-cavity surface-emitting lasers (VCSEL) and _ber-optics borrowed from telecommunication systems to directly transmit analog signals from an SiPM (see Figure 1). Our experiments used 3mm x 3mm x $5 \mathrm{~mm}$ teflon wrapped, and $3 \mathrm{~mm} \times$ 3mm x 20mm ESR wrapped LYSO scintillation crystals, 3mm x 3mm C30742- 33-50-C SiPMs (Excelitas, Waltham, USA), ZX60-4016E+ preamplifiers (Minicircuits, Brooklyn,

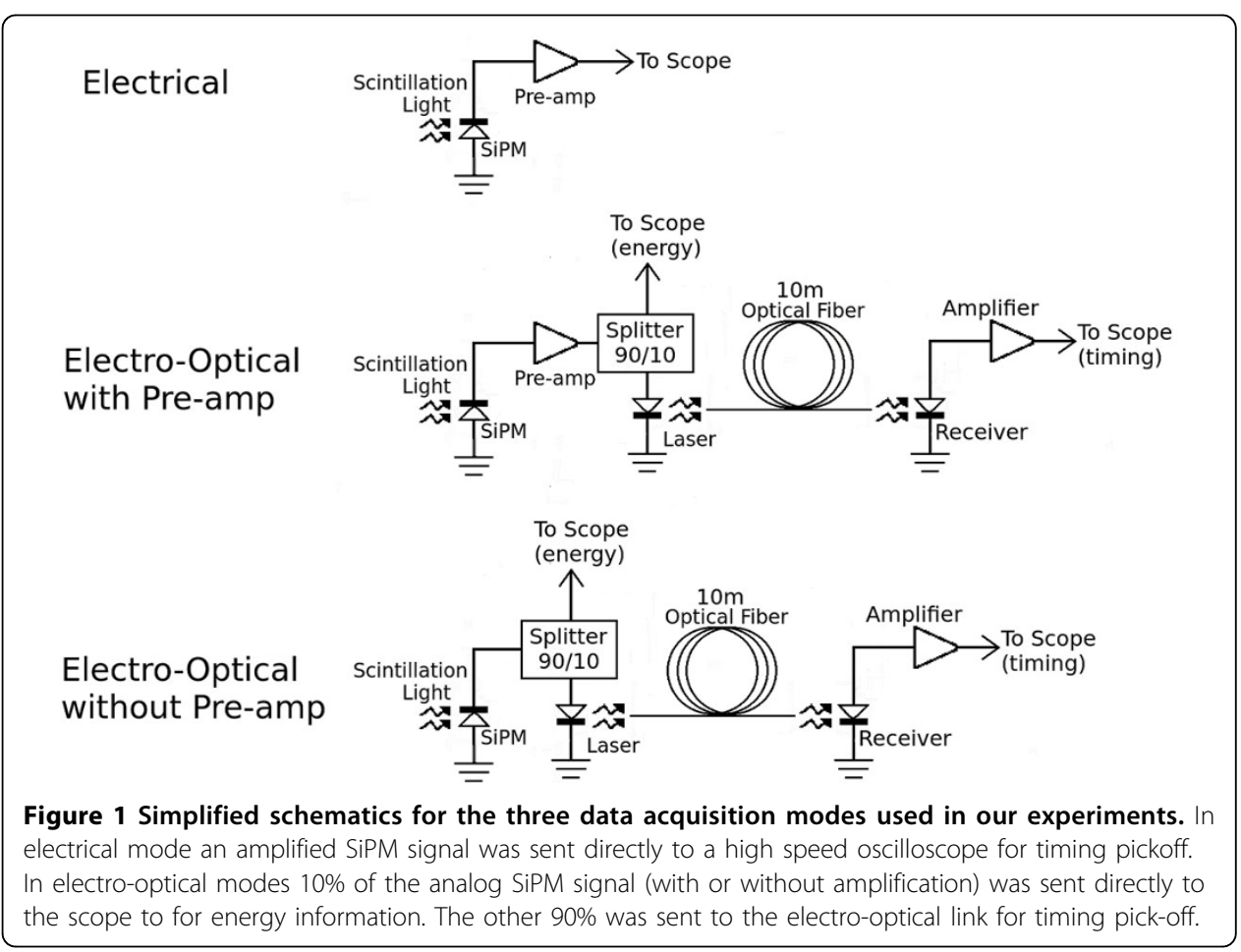

(c) 2014 Bieniosek and Levin; licensee Springer This is an Open Access article distributed under the terms of the Creative Commons Attribution License (http://creativecommons.org/licenses/by/4.0), which permits unrestricted use, distribution, and reproduction in any medium, provided the original work is properly cited. 


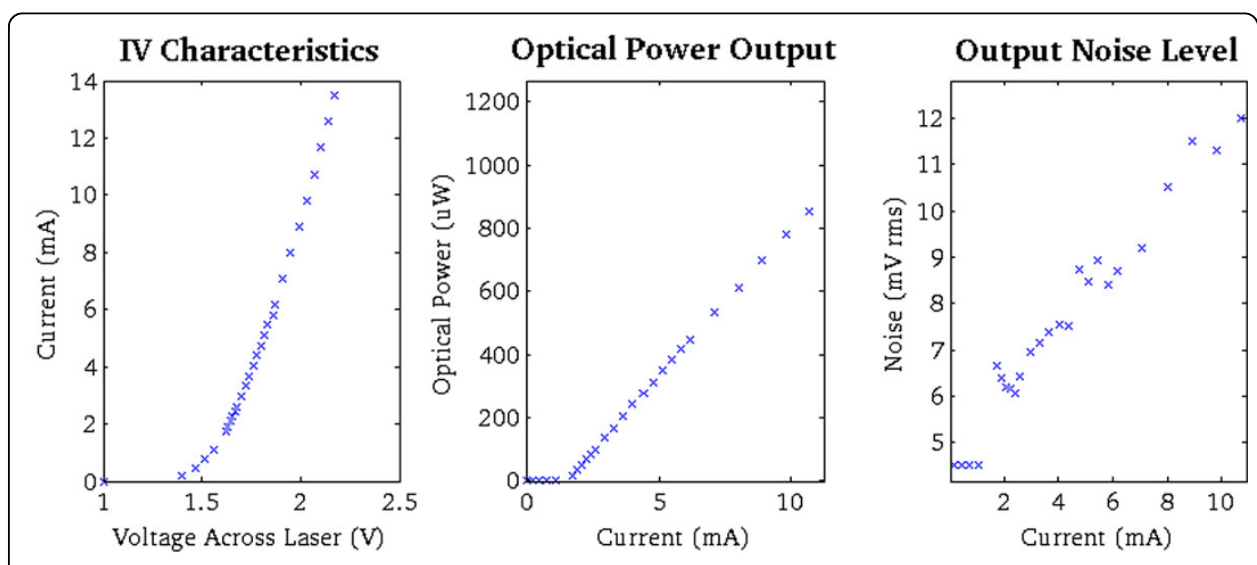

Figure 2 Characterization of the HFE4192-58X VCSEL. Its current rises exponentially with voltage (left). After the laser threshold current is surpassed, it's optical power (middle) and output noise levels (right) rise linearly with current. To optimize SNR a laser bias just above the VCSEL's threshold (1.7mA) was chosen.

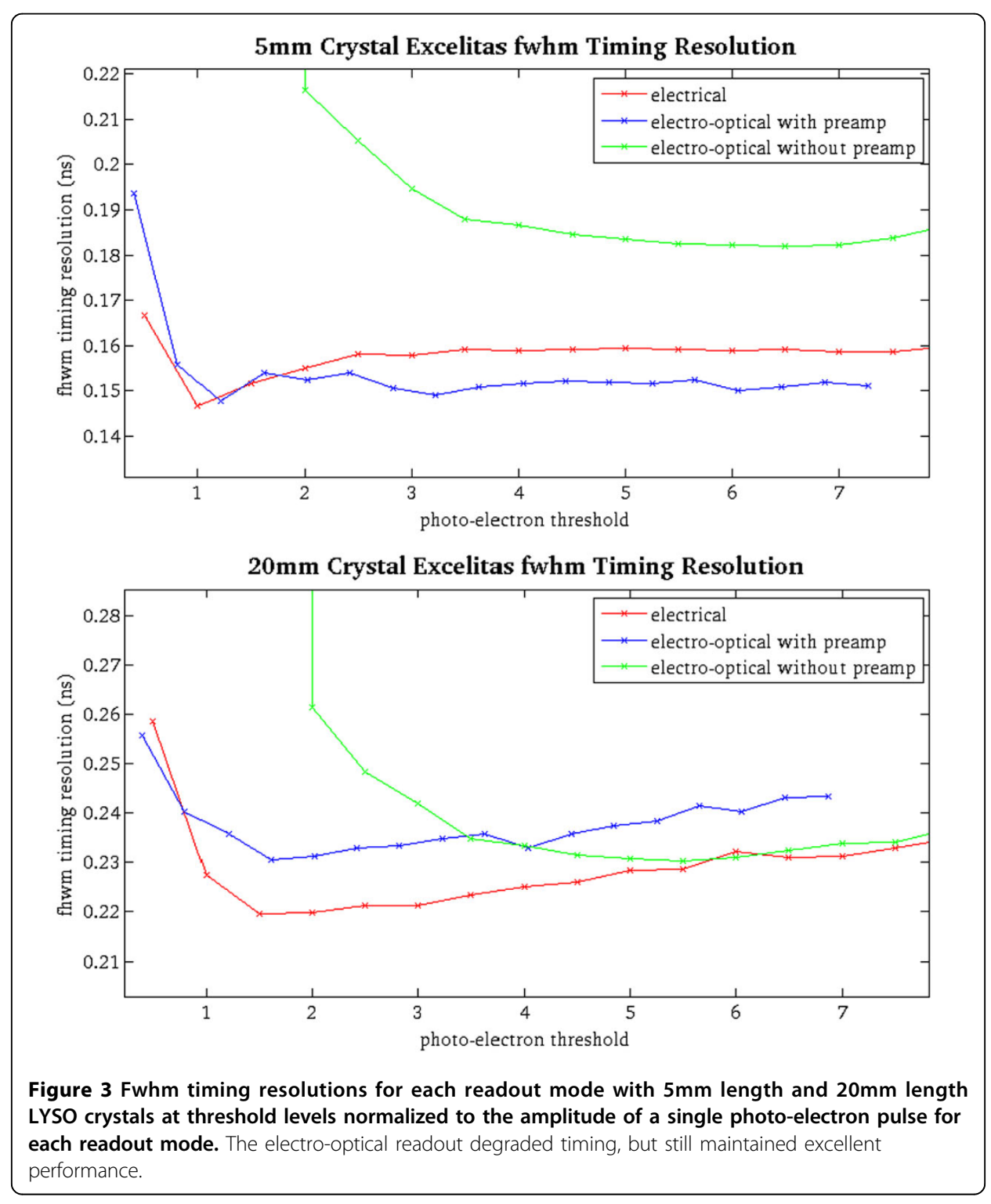


USA), HFE4192-58X VCSELs (Finisar, Sunnyvale,USA), and 10m multi- mode optical patch cables. The VCSEL was characterized as seen in Figure 2. To get the best analog performance a bias current just above the laser threshold $(1.7 \mathrm{~mA})$ was chosen to minimize noise, and power dissipation $(2.8 \mathrm{~mW})$.

The results of the timing experiments are seen in Figure 3. The best timing resolution achieved with $5 \mathrm{~mm}$ length crystals was $147 \mathrm{ps}+/-1 \mathrm{ps}$ in electrical mode, $148 \mathrm{ps}+/-2 \mathrm{ps}$ in electro-optical with preamp mode, and $182+/-2$ ps in electro-optical without preamp mode. With $20 \mathrm{~mm}$ length crystals the best timing achieved was $220+/-3 \mathrm{ps}$ in electrical mode, $230+/$ - 2ps in electro-optical with preamp mode, and $230+/$ - 2ps in electro-optical without preamp mode.

This work shows that SiPM ToF information can be preserved after analog electrooptical transmission. In the future this readout strategy could drastically simplify the design of high performance PET/MRI systems by reducing the in-bore electronics to two active components (SiPMs and VCSELs).

Acknowledgments

The authors would like to thank Joshua Cates for his help in performing timing measurements. This work was supported in part by $\mathrm{NIH}$ research grant R01EB011552 and $\mathrm{NIH}$ training grant 1F31CA171573-01.

\section{Authors' details}

'Department of Radiology, Stanford University, Stanford, CA 94305, USA. ${ }^{2}$ Molecular Imaging Program at Stanford (MIPS), Stanford, CA 94305, USA. ${ }^{3}$ Department of Physics, Stanford University, Stanford, CA 94305, USA. ${ }^{4}$ Department of Electrical Engineering, Stanford University, Stanford, CA 94305, USA. ${ }^{5}$ Department of Bioengineering, Stanford University, Stanford, CA 94305, USA.

Published: 29 July 2014

Reference

1. Olcott PD, et al: Novel Electro-Optically Coupled MR-Compatible PET Detectors. IEEE NSS Conference Record 2008, 4640-4645.

doi:10.1186/2197-7364-1-S1-A12

Cite this article as: Bieniosek and Levin: Analog electro-optical readout of SiPMs for compact, low power ToF PET/MRI. EJNMMI Physics 2014 1(Suppl 1):A12.

\section{Submit your manuscript to a SpringerOpen ${ }^{\odot}$} journal and benefit from:

- Convenient online submission

- Rigorous peer review

- Immediate publication on acceptance

- Open access: articles freely available online

- High visibility within the field

- Retaining the copyright to your article

Submit your next manuscript at $\boldsymbol{\nabla}$ springeropen.com 